\title{
Applications of Swarm Intelligent Algorithm in Economic Model
}

\author{
Shi Yaping \\ Guangdong University of Science and Technology, Guangdong, Dongwan, 523083
}

Keywords: swarm intelligent algorithm; economic model

\begin{abstract}
Swarm intelligent optimization algorithm is a method which completes the optimization algorithm through information interaction between some or the entire individuals based on random search optimization. This paper puts forward the optimization method of micro grid economic model based on the particle algorithm through the research on swarm intelligent algorithm. In the micro grid economic model, this paper calculates the optimal economic dispatch results of micro grid system. The calculation results are compared and analyzed, which has realistic meaning to the design and planning of the micro grid energy management.
\end{abstract}

\section{Swarm Intelligent Algorithm}

\subsection{Brief Introduction}

Swarm intelligent optimization algorithm completes the optimization algorithm through information interaction between some individuals, which includes some typical swarm intelligent algorithms such as particle swarm algorithm, ant colony algorithm, and the emerging of Drosophila algorithm. Swarm intelligent algorithms are based on random search to optimization. They put forward are derived from certain biological phenomena, adaptive to some extent biological. Swarm intelligent algorithm is one of the earliest of the genetic algorithm is proposed to the last century 60's, the United States is by Professor Holland Michigan and put forward university students. With the genetic algorithm in optimization of success, more scholars began attention to nature biological adaptive searching optimal based on the importance and feasibility. In the subsequent proposed particle swarm optimization algorithm, immune algorithm and ant colony algorithm of swarm intelligent optimization algorithm plays a very important role in people's production and life, especially in the field of engineering.

\subsection{Algorithm Principles}

Particle swarm optimization algorithm application principle is very simple: each individual in a population can benefit from the experience of the past approaches of the individual. Particle swarm optimization algorithm is to find the best position of individual particles through the environment to complete the optimization of particle swarm optimization algorithm. The biggest difference between other swarm intelligent algorithms is that each optimization individual is considered as space without volume particle instead of using the optimization of individual operator.

We assume the $\mathrm{i}$ optimal individual is $X_{i}=\left(X_{i 1}, X_{i 2}, \ldots, X_{i D}\right)$. The best place of it is $p_{i}=\left(p_{i 1}, p_{i 2}, \ldots, p_{i D}\right)$, which is recorded as pbest. We use g to record the best position in the group. The particle velocity is $\mathrm{Vi}, V_{i}=\left(V_{i 1}, V_{i 2}, \ldots, V_{i D}\right)$. We have:

$$
\begin{gathered}
v_{i d}^{k}=w v_{i d}^{k-1}+c_{1} \operatorname{rand}_{1}\left(p_{i d}-x_{i d}^{k-1}\right)+c_{2} \operatorname{rand}_{2}\left(p_{g d}-x_{i d}^{k-1}\right) \\
x_{i d}^{k}=x_{i d}^{k-1}+v_{i d}^{k-1}
\end{gathered}
$$

W represents inertia weight, $\mathrm{C} 1$ and $\mathrm{C} 2$ as the acceleration constants, rand1 and rand2 two in arbitrary $[0,1]$ number. In the formula (1) and (2), $\mathrm{m}$ is the total particle number. For the $\mathrm{D}$ component, which is based on the $\mathrm{K}$ iteration individual flight velocity vector appears, is the position vector based component, $\mathrm{P}_{\text {id }}$ for the $\mathrm{D}$ component pbest $_{\mathrm{i}}$, PGD for the $\mathrm{D}$ component of 
gbest. $\mathrm{V}_{\mathrm{i}}$ said the particle velocity, it is limited to a maximum speed of $\mathrm{V}_{\max }$. I particles by type (1) and (2) determine the next motion position.

\subsection{Optimization Steps}

Process optimization of particle swarm optimization algorithm is divided into 6 steps:

Step l: Initialization of the particle swarm, the iteration number is set to $k=0$. In randomly generated particles in the $\mathrm{D}$ dimension of the feasible region, as the initial value of the particle swarm, each particle corresponds to a group of variables selection. At the same time the particle flight speed initialized;

Step 2: According to the optimal value step l, the objective functions of particle's fitness to evaluate;

Step 3: Record the best position of each particle pbest and calculates the best location of the current pbest;

Step 4: On the basis of Step 3, we compare the best position of each particle with the global gbest. If the result is good, we should reset gbest;

Step 5: Adjust the particle's position and speed;

Step 6: Determine whether convergence. If not, return to Step2.

\section{Economic Model of a Typical Micro Grid System}

Microgrid is an overall grid in terms of a large grid. Micro power network in contains a variety of energy resources for power generation, including renewable energy power generation, such as wind power, solar photovoltaic power generation, but also save non-renewable energy power generation, such as micro gas turbine, fuel tank. A typical microgrid system includes an electrical load and the microgrid control system three parts with the distributed power. Distributed power consists of photovoltaic power generation, wind power generation and energy storage system, phase electrical load regardless of power load, the important load and indoor Sichuan Electric class three. The microgrid control system can manage its internal distributed power supply and load. In the event of failure, the microgrid seamlessly switches to solitary station operation mode. In this mode, the power distribution need be out of operation and continue to maintain a negative power.

When the microgrid operation, if the PV and wind power generating capacity is larger than the micro correction within the load, will be redundant power storage to storage systems, photovoltaic and wind power if reduced or no output, you can release the energy storage towel. Part of the electric power element. When the microgrid islanded operation, through the energy storage system for charging and discharging control, can realize the distributed generation system with micro real-time balancing network load, so as to ensure the stable operation of micro solitary station. A very important part in the Multi-agent system is the communication. The communication mode of Multi-agent system is the system of share transfer in the news. Communication protocol consists of interactive network transmission protocols, and the high-level communication constraint. The following requirements on the communication language system with simple form and easy to understand, is interchangeable with other systems. There is a hierarchy, semantic grammar specification in it.

The main task of micro grid economic dispatching is will be mainly involved in solar and wind will be the optimal allocation, the cost of power generation and to the load center power network loss is the comprehensive index of the reference standard, seek the power supply configuration. The prerequisite is to meet the load growth and safe and reliable power supply to achieve the most economic operation of micro grid. The combination of microgrid economic index optimization, process scheduling economy of micro grid is: the main control Agent with various micro sources Agent and the load of Agent, grid connected communication. During the operation of micro grid system, the scheduling process is: first load of Agent through the information transmission to the main control Agent send load demand; and then the main control Agent to each micro source Agent sends bidding broadcasting; each micro source Agent quotation to the main control Agent according to the present situation; the main control Agent were pooled according to each micro source 
submitted price, establish good substitution algorithm model of calculation, get the bidding result. Finally, each micro source Agent, according to the main Agent, gives the auction results to adjust their investment capacity.

\section{Applications of Swarm Intelligent Algorithm in Economic Model}

\subsection{Optimization Process}

This paper on the micro grid modeling and optimization process is complex. The particle swarm algorithm constructs a single particle of overall economic according to the specific situation. The process of optimization, the Agent common multiple dimension optimization calculation, the optimal single particle values and group values. The optimization process is as follows:

Step l: Initialization of the particle swarm, the iteration number is set to $k=100.100 / 500$ a randomly generated particles in the 5 dimension of the feasible region, as the initial particle swarm $\mathrm{X}$ value, initial value for each particle corresponds to the particle flight speed, at the same time, $\mathrm{V}$ is initialized;

Step 2: According to the optimal value step l, the objective function of the first generation of the first particle's fitness is calculated to obtain the optimal particle, values, and so on, each particle was optimized, and then calculate the economic index of 4 Agent $\mathrm{f}(\mathrm{x})$;

Step 3: Calculate the global first generation 100 particles in optimal $\mathrm{f}(\mathrm{X})$ value;

Step 4: The first generation of the initial population optimization process substitution carries on 100 times of iteration;

Step 5; Pbest will be the best position of each particle down record, the best current position;

Step 6: On the basis of Step5 comparison, the best position of each particle and the global gbest. If good, then reset the gbest.

\subsection{Simulation Results}

According to the above process, we can achieve the following results:

When the population size is 100 ; load periods is selected 9; load demand is $49 \mathrm{KW}$, the output of the various units of the results shown in the table below:

Table 1: Results of population size 100 and load periods is 9

\begin{tabular}{|c|c|c|c|c|c|c|}
\hline Agent1 & Agent2 & Agent3 & Agent4 & size & period & $\mathrm{f}(\mathrm{x})$ \\
\hline 18.57 & 13.41 & 23.6 & 19.24 & 100 & 9 & 30.3 \\
\hline
\end{tabular}

When the population size is 500 ; load periods is selected 9; load demand is $47 \mathrm{KW}$, the output of the various units of the results shown in the table below:

Table 2: Results of population size 500 and load periods is 9

\begin{tabular}{|c|c|c|c|c|c|c|}
\hline Agent1 & Agent2 & Agent3 & Agent4 & size & period & $\mathrm{f}(\mathrm{x})$ \\
\hline 10.2 & 14.56 & 15.26 & 10.57 & 500 & 9 & 33.19 \\
\hline
\end{tabular}

When the population size is 100 ; load periods is selected 10; load demand is $55 \mathrm{KW}$, the output of the various units of the results shown in the table below:

Table 3: Results of population size 500 and load periods is 10

\begin{tabular}{|c|c|c|c|c|c|c|}
\hline Agent1 & Agent2 & Agent3 & Agent4 & size & period & $\mathrm{f}(\mathrm{x})$ \\
\hline 19.93 & 9.91 & 16.02 & 10.21 & 100 & 10 & 38.32 \\
\hline
\end{tabular}

\subsection{Results Analysis}

Particle swarm optimization algorithm of economic parameters of population size for the two parameters 100 and 500 finds the comparison chart of optimal algorithm based on various optimizations called population scale frame. The effect can be seen economic optimizations under different scales are different. In general population scale, the condition of the smaller population optimization is better, but not stable. 
Through the particle swarm optimization of population size in power optimization results contrast between 100 and 500 under two kinds of situations, we can see that the larger the population size is, the smaller the power optimization effect is.

\section{References}

[1] Chen Zhuo, Liu Xiangshuang. A new-style clustering algorithm based on swarm intelligent theory [J]. Journal of Harbin Institute of Technology, 2007,01

[2] Haiyan Zhao, Zhili Pei, Jingqing Jiang, A Hybrid Swarm Intelligent Method Based on Genetic Algorithm and Artificial Bee Colony,2010,03

[3] Yuan Ping, Wang Hao. Research on particle swarm algorithm of intelligent optimization [J]Information Technology, 2007,07

[4] Liu Xiangshuang, Xu Jianliang.Multi-Agent Clustering Algorithm Based on Swarm Intelligent Theory [J]. Application Research of Computers, 2012,02 